\title{
Approximation of an optimal control problem in coefficient for variational inequality with anisotropic $p$-Laplacian
}

\author{
Olha P. Kupenko and Rosanna Manzo
}

\begin{abstract}
We study an optimal control problem for a variational inequality with the so-called anisotropic $p$-Laplacian in the principle part of this inequality. The coefficients of the anisotropic $p$-Laplacian, the matrix $A(x)$, we take as a control. The optimal control problem is to minimize the discrepancy between a given distribution $y_{d} \in L^{2}(\Omega)$ and the solutions $y \in K \subset W_{0}^{1, p}(\Omega)$ of the corresponding variational inequality. We show that the original problem is well-posed and derive existence of optimal pairs. Since the anisotropic $p$-Laplacian inherits the degeneracy with respect to unboundedness of the term $\left|(A(x) \nabla y, \nabla y)_{\mathbb{R}^{N}}\right|^{\frac{p-2}{2}}$, we introduce a two-parameter model for the relaxation of the original problem. Further we discuss the asymptotic behavior of relaxed solutions and show that some optimal pairs to the original problem can be attained by the solutions of two-parametric approximated optimal control problems.
\end{abstract}

Mathematics Subject Classification. Primary 35J70, 49J20, 49J45; Secondary $93 \mathrm{C} 73$.

Keywords. Anisotropic p-Laplacian, Optimal control, Control in coefficients, Variational inequality.

\section{Introduction}

In this paper we deal with the following optimal control problem (OCP) in coefficients for variational inequality

$$
\left\{\begin{array}{l}
\text { Minimize } I(A, y)=\left\|y-y_{d}\right\|_{L^{2}(\Omega)}^{2} \text { subject to the constraints } \\
\left\langle-\Delta_{p}(A, y), v-y\right\rangle_{W^{-1, q}(\Omega) ; W_{0}^{1, p}(\Omega)} \geq \int_{\Omega} f(v-y) d x \quad \text { for all } v \in K, \\
A \in \mathfrak{A}_{a d},
\end{array}\right.
$$


where

$$
-\Delta_{p}(A, y)=-\operatorname{div}\left(|(A \nabla y, \nabla y)|^{\frac{p-2}{2}} A \nabla y\right)
$$

is the anisotropic $p$-Laplacian, $p$ satisfies $2 \leq p<+\infty, K$ is a nonempty convex closed subset of the space $W_{0}^{1, p}(\Omega)$, the symmetric matrix of anisotropy $A \in L^{\infty}\left(\Omega ; R^{N \times N}\right)$ is taken as a control, $y_{d} \in L^{2}(\Omega)$ and $f \in L^{2}(\Omega)$ are given distributions, and $\mathfrak{A}_{a d}$ denotes the class of admissible controls which will be specified later on (see (2.2), (2.3)).

The interest to variational inequalities whose principle part is an anisotropic $p$-Laplace-like operator arises from various applied contexts related to composite materials such as nonlinear dielectric composites, whose nonlinear behavior is modeled by the so-called power low (see, for instance, $[3,15]$ and references therein). It is sufficient to say that anisotropic $p$-Laplacian $\Delta_{p}(A, y)$ has profound background both in the theory of anisotropic and nonhomogeneous media and in Finsler or Minkowski geometry [19]. As a rule, the effect of anisotropy appears naturally in a wide class of geometry-Finsler geometry. A typical and important example of Finsler geometry is Minkowski geometry. In this case, anisotropic Laplacian is closely related to a convex hypersurface in $\mathbb{R}^{N}$, which is called the Wulff shape [18]. Since the topology of the Wulff shape essentially depends on the matrix of anisotropy $A(x)$, it is reasonable to take such matrix as a control. From mathematical point of view, the interest of anisotropic $p$-Laplacian lies on its nonlinearity and an effect of degeneracy, which turns out to be the major difference from the standard Laplacian on $\mathbb{R}^{N}$.

Using the direct method in the Calculus of Variations, we show in Sect. 3 that the optimal control problem (1.1) has a nonempty set of solutions provided the admissible controls $A(x)$ are uniformly bounded in $B V$-norm, in spite of the fact that the corresponding quasilinear differential operator - $\operatorname{div}\left(|(A \nabla y, \nabla y)|^{\frac{p-2}{2}} A \nabla y\right)$, in principle, has degeneracies as $\left|A^{\frac{1}{2}} \nabla y\right|$ tends to zero [2]. Moreover, when the term $|(A \nabla y, \nabla y)|^{\frac{p-2}{2}}$ is regarded as the coefficient of the Laplace operator, we have the case of unbounded coefficients (see $[9,10])$. In order to avoid degeneracy with respect to the control $A(x)$, we assume that matrix $A(x)$ has a uniformly bounded spectrum away from zero. As for the optimal control problems in coefficients for degenerate elliptic equations and variational inequalities, we can refer to $[4,7,8,12-14]$.

A number of regularizations have been suggested in the literature. See [17] for a discussion for what has come to be known as $(\varepsilon, p)$-Laplace problem, such as $-\operatorname{div}\left(\left(\varepsilon+|\nabla y|^{2}\right)^{\frac{p-2}{2}} \nabla y\right)$. While the $(\varepsilon, p)$-Laplacian regularizes the degeneracy as the gradients tend to zero, the term $|\nabla y|^{p-2}$, viewed again as a coefficient, may grow large [5]. Therefore, following ideas of [6], we introduce yet another regularization that leads to a sequence of monotone and bounded approximations $\mathcal{F}_{k}\left(\left|A^{\frac{1}{2}} \nabla y\right|^{2}\right)$ of $\left|A^{\frac{1}{2}} \nabla y\right|^{2}$. As a result, for fixed parameter $p \in[2, \infty)$ and control $A(x)$, we arrive at a two-parameter variational problem governed by operator $-\operatorname{div}\left(\left(\varepsilon+\mathcal{F}_{k}\left(\left|A^{\frac{1}{2}} \nabla y\right|^{2}\right)\right)^{\frac{p-2}{2}} A \nabla y\right)$. Finally, we deal with a two-parameter family of optimal control problems in the coefficients for monotone nonlinear variational inequalities. We consequently provide the 
well-posedness analysis for the perturbed elliptic variational inequalities as well as for the optimal control problem in Sects. 4 and 5. In particular, we show in Sect. 5 that the solutions of two-parametric family of perturbed optimal control problems can be considered as appropriate approximations to optimal pairs for the original problem (1.1) (see Theorem 5.2). To the end, we note that the approximation and regularization are not only considered to be useful for the mathematical analysis, but also for the purpose of numerical simulations. The numerical analysis as well as the case of degenerating controls are subjects to future publications.

\section{Setting of the optimal control problem}

Let $\Omega$ be a bounded open subset of $\mathbb{R}^{N}(N \geq 1)$ with a Lipschitz boundary. Let $p$ be a real number such that $2 \leq p<\infty$, and let $q=p /(p-1)$ be the conjugate of $p$. Let $\mathbb{S}^{N}:=\mathbb{R}^{\frac{N(N+1)}{2}}$ be the set of all symmetric matrices $A=\left[a_{i j}\right]_{i, j=1}^{N}$, $\left(a_{i j}=a_{j i} \in \mathbb{R}\right)$. We suppose that $\mathbb{S}^{N}$ is endowed with the Euclidian scalar product $A \cdot B=\operatorname{tr}(A B)=a_{i j} b_{i j}$ and with the corresponding Euclidian norm $\|A\|_{\mathbb{S}^{N}}=(A \cdot A)^{1 / 2}$. We also make use of the so-called spectral norm $\|A\|_{2}:=$ $\sup \left\{|A \xi|: \xi \in \mathbb{R}^{N}\right.$ with $\left.|\xi|=1\right\}$ of matrices $A \in \mathbb{S}^{N}$, which is different from the Euclidean norm $\|A\|_{\mathbb{S}^{N}}$. However, the relation $\|A\|_{2} \leq\|A\|_{\mathbb{S}^{N}} \leq \sqrt{N}\|A\|_{2}$ holds true for all $A \in \mathbb{S}^{N}$.

Let $L^{1}(\Omega)^{\frac{N(N+1)}{2}}=L^{1}\left(\Omega ; \mathbb{S}^{N}\right)$ be the space of integrable functions whose values are symmetric matrices. By $B V\left(\Omega ; \mathbb{S}^{N}\right)$ we denote the space of all matrices in $L^{1}\left(\Omega ; \mathbb{S}^{N}\right)$ for which the norm

$$
\begin{aligned}
& \|C\|_{B V\left(\Omega ; \mathbb{S}^{N}\right)}=\|C\|_{L^{1}\left(\Omega ; \mathbb{S}^{N}\right)}+\int_{\Omega}|D C|=\|C\|_{L^{1}\left(\Omega ; \mathbb{S}^{N}\right)} \\
& \quad+\sum_{1 \leq i \leq j \leq N} \sup \left\{\int_{\Omega} c_{i j} \operatorname{div} \varphi d x: \varphi \in C_{0}^{1}\left(\Omega ; \mathbb{R}^{N}\right),|\varphi(x)| \leq 1 \text { for } x \in \Omega\right\}
\end{aligned}
$$

is finite.

Let $\xi_{1}, \xi_{2}$ be given elements of $L^{\infty}(\Omega) \cap B V(\Omega)$ satisfying the conditions

$$
0<\alpha \leq \xi_{1}(x) \leq \xi_{2}(x) \text { a.e. in } \Omega,
$$

where $\alpha$ is a given positive value. Let $y_{d} \in L^{2}(\Omega)$ and $f \in L^{2}(\Omega)$ be given distributions. We define the class of admissible controls $\mathfrak{A}_{a d}$ as follows

$$
\mathfrak{A}_{a d}=\left\{\begin{array}{ll}
A \in L^{1}\left(\Omega ; \mathbb{S}^{N}\right) & \begin{array}{l}
\xi_{1}^{2}\|\eta\|^{2} \leq(\eta, A \boldsymbol{\eta}) \leq \xi_{2}^{2}\|\eta\|^{2} \text { a.e. in } \Omega \forall \eta \in \mathbb{R}^{N} \\
A^{\frac{1}{2}} \in B V\left(\Omega ; \mathbb{S}^{N}\right), \int_{\Omega}\left|D A^{\frac{1}{2}}\right| \leq \gamma
\end{array}
\end{array}\right\},
$$

where $\gamma>0$ is a given constant. In view of estimates

$$
\begin{gathered}
\left\|A^{\frac{1}{2}}(x)\right\|_{\mathbb{S}^{N}} \leq \sqrt{N}\left\|A^{\frac{1}{2}}(x)\right\|_{2} \leq \sqrt{N} \xi_{2}(x) \quad \text { a.e. in } \Omega \\
\int_{\Omega}\|A\|_{\mathbb{S}^{N}}^{\frac{p}{2}} d x \leq \int_{\Omega}\left\|A^{\frac{1}{2}}\right\|_{\mathbb{S}^{N}}^{p} d x \leq \int_{\Omega}\left\|A^{\frac{1}{2}}\right\|_{\mathbb{S}^{N}}^{p-1}\left\|A^{\frac{1}{2}}\right\|_{\mathbb{S}^{N}} d x
\end{gathered}
$$




$$
\leq N^{\frac{p-1}{2}}\left\|\xi_{2}\right\|_{L^{\infty}(\Omega)}^{p-1} \int_{\Omega}\left\|A^{\frac{1}{2}}\right\|_{\mathbb{S}^{N}} d x
$$

it is clear that $\mathfrak{A}_{a d}$ is a nonempty convex subset of $L^{\frac{p}{2}}\left(\Omega ; \mathbb{S}^{N}\right)$ with empty topological interior.

Let $K$ be a nonempty convex closed subset of the space $W_{0}^{1, p}(\Omega)$ such that $0 \in K$. The optimal control problem we consider in this paper is to minimize the discrepancy between the distribution $y_{d} \in L^{2}(\Omega)$ and the solutions $y \in K$ of the following variational inequality

$$
\left\langle-\Delta_{p}(A, y), v-y\right\rangle_{W^{-1, q}(\Omega) ; W_{0}^{1, p}(\Omega)} \geq \int_{\Omega} f(v-y) d x \quad \text { for all } v \in K,
$$

by choosing an appropriate matrix-valued function $A \in \mathfrak{A}_{a d}$ as control. Here, $\Delta_{p}(A, \cdot): W_{0}^{1, p}(\Omega) \rightarrow W^{-1, q}(\Omega)$ is the so-called anisotropic $p$-Laplacian which usually can be defined by the rule (see [2] and references therein)

$$
\Delta_{p}(A, y)=\operatorname{div}\left(|(A \nabla y, \nabla y)|^{\frac{p-2}{2}} A \nabla y\right)
$$

and $f \in L^{2}(\Omega)$ is a given distribution.

Let us denote by $\Xi$ the set of admissible solutions to problem (1.1), i.e.

$$
\Xi=\left\{(A, y) \mid A \in \mathfrak{A}_{a d}, y \in K \subset W_{0}^{1, p}(\Omega),(A, y) \text { are related by }(2.13)\right\}
$$

Definition 2.1. We say that a pair $\left(A^{0}, y^{0}\right) \in B V\left(\Omega ; \mathbb{S}^{N}\right) \times W_{0}^{1, p}(\Omega)$ is an optimal solution to problem (1.1) if

$$
\left(A^{0}, y^{0}\right) \in \Xi \quad \text { and } \quad I\left(A^{0}, y^{0}\right)=\inf _{(A, y) \in \Xi} I(A, y) .
$$

The existence of a unique solution to the variational inequality (2.4) follows from an abstract well-known theorem on monotone operators (see [16, Theorems 8.2, 8.3, 8.4]).

Theorem 2.2. Let $V$ be a reflexive separable Banach space and $K \subset V$ be a nonempty convex closed subset. Let $V^{*}$ be the dual space, and let $\mathcal{A}: K \rightarrow V^{*}$ be a bounded, semicontinuous, strictly monotone operator and coercive in the following sense

$$
\text { there exists } v_{0} \in K \text { such that } \lim _{\|y\|_{V \rightarrow \infty}} \frac{\left\langle\mathcal{A} y, y-v_{0}\right\rangle_{V^{*} ; V}}{\|y\|_{V}}=+\infty \text {. }
$$

Then the variational inequality

$$
\text { find } y \in K \text { s.t. }\langle\mathcal{A} y, v-y\rangle_{V^{*} ; V} \geq\langle f, v-y\rangle_{V^{*} ; V}, \quad \forall v \in K,
$$

has a unique solution for each $f \in V^{*}$. Moreover, the variational inequality (2.7) is equivalent to the following one

find $y \in K$ s.t. $\langle\mathcal{A} v, v-y\rangle_{V^{*} ; V} \geq\langle f, v-y\rangle_{V^{*} ; V} \quad$ for all $v \in K$.

We recall that operator $A$ is called

- monotone if

$$
\langle\mathcal{A} y-\mathcal{A} v, y-v\rangle_{V^{*} ; V} \geq 0, \quad \forall y, v \in V
$$


- strictly monotone if (2.9) holds and

$$
\langle\mathcal{A} y-\mathcal{A} v, y-v\rangle_{V^{*} ; V}=0 \Longrightarrow y=v ;
$$

- semicontinuous, if

the function $\mathbb{R} \ni t \mapsto\langle\mathcal{A}(y+t v), w\rangle_{V^{*} ; V}$ is continuous for all $y, v, w \in V$.

Having defined operator $\mathcal{A}$ as a mapping $W_{0}^{1, p}(\Omega) \rightarrow W^{-1, q}(\Omega)$ by the rule

$$
\begin{aligned}
\langle\mathcal{A} \varphi, v\rangle_{W^{-1, q}(\Omega) ; W_{0}^{1, p}(\Omega)} & :=\int_{\Omega}|(A \nabla \varphi, \nabla \varphi)|^{\frac{p-2}{2}}(A \nabla \varphi, \nabla v) d x \\
& =\int_{\Omega}\left|A^{\frac{1}{2}} \nabla \varphi\right|^{p-2}(A \nabla \varphi, \nabla v) d x,
\end{aligned}
$$

it is easy to see that in this case we have $\mathcal{A}=-\Delta_{p}(A, \cdot)$. Let us show that operator $\mathcal{A}$ satisfies all assumptions of Theorem 2.2 for each $A \in \mathfrak{A}_{a d}$. Indeed, the right-hand side of $(2.12)$ is continuous in $v \in W_{0}^{1, p}(\Omega)$ and, therefore, represents an element of $W^{-1, q}(\Omega)$ because

$$
\begin{aligned}
& \int_{\Omega}\left|A^{\frac{1}{2}} \nabla \varphi\right|^{p-2}(A \nabla \varphi, \nabla v) d x \leq\left(\int_{\Omega}\left|A^{\frac{1}{2}} \nabla \varphi\right|^{p} d x\right)^{\frac{p-1}{p}}\left(\int_{\Omega}\left|A^{\frac{1}{2}} \nabla v\right|^{p} d x\right)^{\frac{1}{p}} \\
& \quad \leq\left\|\xi_{2}\right\|_{L^{\infty}(\Omega)}^{p}\|\nabla \varphi\|_{L^{p}(\Omega)^{N}}^{p-1}\|\nabla v\|_{L^{p}(\Omega)^{N}}=\left\|\xi_{2}\right\|_{L^{\infty}(\Omega)}^{p}\|\varphi\|_{W_{0}^{1, p}(\Omega)}^{p-1}\|v\|_{W_{0}^{1, p}(\Omega)}
\end{aligned}
$$

(we apply here Hölder's inequality and the estimate $\left|A^{\frac{1}{2}} \nabla \varphi\right|^{p} \leq \xi_{2}^{p}|\nabla \varphi|^{p}$ coming from the condition $\left.A \in \mathfrak{A}_{a d}\right)$. Hence, operator $\mathcal{A}: W_{0}^{1, p}(\Omega) \rightarrow W^{-1, q}(\Omega)$ is bounded. In order to prove the coercivity of $\mathcal{A}$, we set $v_{0}=0 \in K$ in (2.6) and get the desired property immediately, since

$$
\langle\mathcal{A} y, y\rangle_{W^{-1, q}(\Omega) ; W_{0}^{1, p}(\Omega)} \geq \alpha^{p}\|y\|_{W_{0}^{1, p}(\Omega)}^{p} .
$$

As for the proof of strict monotonicity and semicontinuity of operator $\mathcal{A}$, we refer for the details to $[16,17])$.

Thus, according to Theorem 2.2, variational inequality (2.4) is equivalent to the following one

$$
\int_{\Omega}|(A \nabla v, \nabla v)|^{\frac{p-2}{2}}(A \nabla v, \nabla v-\nabla y) d x \geq \int_{\Omega} f(v-y) d x, \quad \forall v \in K
$$

and it admits a unique solution $y \in K$ for every admissible control $A \in \mathfrak{A}_{a d}$.

Taking this fact into account, we state that the set of admissible pairs $\Xi$ to problem (1.1) is nonempty.

Remark 2.3. It is easy to see that the set of solutions $\{y=y(A) \in K: A \in$ $\left.\mathfrak{A}_{a d}\right\}$ to problem (2.4) or (2.13) is bounded in $W_{0}^{1, p}(\Omega)$. Indeed, since the set $K \subset W_{0}^{1, p}(\Omega)$ contains zero, we have

$$
\begin{aligned}
\alpha^{p}\|y\|_{W_{0}^{1, p}(\Omega)}^{p} & \leq \int_{\Omega}\left|A^{\frac{1}{2}} \nabla y\right|^{p} d x=\langle\mathcal{A} y, y\rangle \stackrel{\text { by } \stackrel{(2.4)}{\leq}\langle f, y\rangle}{ } \\
& =\int_{\Omega} f y d x \leq\|f\|_{L^{q}(\Omega)}\|y\|_{W_{0}^{1, p}(\Omega)} \leq|\Omega|^{\frac{p-2}{2 p}}\|f\|_{L^{2}(\Omega)}\|y\|_{W_{0}^{1, p}(\Omega)} .
\end{aligned}
$$


Hence, the following estimate for the solutions of (2.4) takes place

$$
\|y\|_{W_{0}^{1, p}(\Omega)} \leq \alpha^{-q}|\Omega|^{\frac{p-2}{2 p(p-1)}}\|f\|_{L^{2}(\Omega)}^{\frac{q}{p}}, \quad \forall A \in \mathfrak{A}_{a d} .
$$

Remark 2.4. Let us set $K^{*}=K \cap B_{R}$, where by $B_{R} \subset W_{0}^{1, p}(\Omega)$ we denote a fixed closed ball centered at zero with a radius $R>\alpha^{-q}|\Omega|^{\frac{p-2}{2 p(p-1)}}\|f\|_{L^{2}(\Omega)}^{\frac{q}{p}}$ (see (2.14)). It is easy to see that $K^{*} \subseteq K$ is a bounded convex closed subset of $W_{0}^{1, p}(\Omega)$ and $y \in K$ is a solution to variational inequality (2.4) (or (2.13)) if and only if $y \in K^{*}$ and

$$
\int_{\Omega}|(A \nabla v, \nabla v)|^{\frac{p-2}{2}}(A \nabla v, \nabla v-\nabla y) d x \geq \int_{\Omega} f(v-y) d x, \quad \forall v \in K^{*} .
$$

Indeed, if $y \in K$ is a solution to (2.13), then, due to estimate (2.14), we have $y \in B_{R}$ and (2.15) obviously holds. To prove the inverse assertion let us suppose by contraposition that $y \in K^{*},(2.15)$ holds true, and there exists an element $z \in K$ such that $z \neq y$ and $z$ is a solution to (2.4). Then $z$ is a solution to variational inequality (2.15) as well, and in view of its uniqueness, we obviously get $y=z$.

\section{Existence of optimal solutions}

In this section we focus on the solvability of optimal control problem (1.1). Hereinafter, we suppose that the space $B V\left(\Omega ; \mathbb{S}^{N}\right) \times W_{0}^{1, p}(\Omega)$ is endowed with the norm $\|(A, y)\|_{B V\left(\Omega ; \mathbb{S}^{N}\right) \times W_{0}^{1, p}(\Omega)}:=\|A\|_{B V\left(\Omega ; \mathbb{S}^{N}\right)}+\|y\|_{W_{0}^{1, p}(\Omega)}$.

Remark 3.1. We recall that a sequence $\left\{f_{k}\right\}_{k=1}^{\infty}$ converges weakly* to $f$ in $B V(\Omega)$ if and only if two following conditions hold (see [1]): $f_{k} \rightarrow f$ strongly in $L^{1}(\Omega)$ and $D f_{k} \stackrel{*}{\rightarrow} D f$ weakly* in the space of Radon measures $\mathcal{M}\left(\Omega ; \mathbb{R}^{N}\right)$. Moreover, if $\left\{f_{k}\right\}_{k=1}^{\infty} \subset B V(\Omega)$ converges strongly to some $f$ in $L^{1}(\Omega)$ and satisfies $\sup _{k \in \mathbb{N}} \int_{\Omega}\left|D f_{k}\right|<+\infty$, then (see, for instance, [1])

(i) $f \in B V(\Omega)$ and $\int_{\Omega}|D f| \leq \liminf _{k \rightarrow \infty} \int_{\Omega}\left|D f_{k}\right|$;

(ii) $f_{k} \stackrel{*}{\rightarrow} f$ in $B V(\Omega)$.

We begin with the following property.

Lemma 3.2. Let $\left\{\left(A_{k}, y_{k}\right) \in \Xi\right\}_{k \in \mathbb{N}}$ be a sequence such that $A_{k}^{\frac{1}{2}} \stackrel{*}{\rightarrow} A^{\frac{1}{2}}$ in $B V\left(\Omega ; \mathbb{S}^{N}\right)$ and $y_{k} \rightarrow y$ in $W_{0}^{1, p}(\Omega)$. Then

$$
\begin{aligned}
& \lim _{k \rightarrow \infty} \int_{\Omega}\left|\left(\nabla \varphi, A_{k} \nabla \varphi\right)\right|^{\frac{p-2}{2}}\left(\nabla y_{k}, A_{k} \nabla \varphi\right) d x \\
& \quad=\int_{\Omega}|(\nabla \varphi, A \nabla \varphi)|^{\frac{p-2}{2}}(\nabla y, A \nabla \varphi) d x, \quad \forall \varphi \in C_{0}^{\infty}(\Omega) .
\end{aligned}
$$

Proof. Since $A_{k}^{\frac{1}{2}} \rightarrow A^{\frac{1}{2}}$ in $L^{1}\left(\Omega ; \mathbb{S}^{N}\right)$ and $\left\{A_{k}^{\frac{1}{2}}\right\}_{k \in \mathbb{N}}$ is bounded in $L^{\infty}\left(\Omega ; \mathbb{S}^{N}\right)$, by Lebesgue's Theorem we get that $A_{k}^{\frac{1}{2}} \rightarrow A^{\frac{1}{2}}$ strongly in $L^{r}\left(\Omega ; \mathbb{S}^{N}\right)$ for every 
$1 \leq r<+\infty$. Hence, $A_{k}^{\frac{1}{2}} \nabla \varphi \rightarrow A^{\frac{1}{2}} \nabla \varphi$ strongly in $L^{p}(\Omega)^{N}$ for every $\varphi \in$ $C_{0}^{\infty}(\Omega)$. Therefore,

$$
\left|A_{k}^{\frac{1}{2}} \nabla \varphi\right|^{p-2} A_{k}^{\frac{1}{2}} \nabla \varphi \rightarrow\left|A^{\frac{1}{2}} \nabla \varphi\right|^{p-2} A^{\frac{1}{2}} \nabla \varphi \quad \text { in } \quad L^{q}(\Omega)^{N}, \quad \forall \varphi \in C_{0}^{\infty}(\Omega) .
$$

Moreover, since $A_{k}^{\frac{1}{2}} \nabla \psi \rightarrow A^{\frac{1}{2}} \nabla \psi$ strongly in $L^{q}(\Omega)^{N}$ for every $\psi \in$ $C_{0}^{\infty}(\Omega)$ and $\nabla y_{k} \rightarrow \nabla y$ in $L^{p}(\Omega)^{N}$, it follows that

$$
\begin{aligned}
\int_{\Omega}\left(A_{k}^{\frac{1}{2}} \nabla y_{k}, \nabla \psi\right) d x & =\int_{\Omega}\left(\nabla y_{k}, A_{k}^{\frac{1}{2}} \nabla \psi\right) d x \rightarrow \int_{\Omega}\left(\nabla y, A^{\frac{1}{2}} \nabla \psi\right) d x \\
& =\int_{\Omega}\left(A^{\frac{1}{2}} \nabla y, \nabla \psi\right) d x, \quad \forall \psi \in C_{0}^{\infty}(\Omega)
\end{aligned}
$$

as a product of weakly and strongly convergent sequences in $L^{p}(\Omega)^{N}$ and $L^{q}(\Omega)^{N}$, respectively. Using the fact that

$$
\sup _{k \in \mathbb{N}}\left\|A_{k}^{\frac{1}{2}} \nabla y_{k}\right\|_{L^{p}(\Omega)^{N}} \leq\left\|\xi_{2}\right\|_{L^{\infty}(\Omega)} \sup _{k \in \mathbb{N}}\left\|\nabla y_{k}\right\|_{L^{p}(\Omega)^{N}}<+\infty,
$$

we finally get from $(3.4)$

$$
A_{k}^{\frac{1}{2}} \nabla y_{k} \rightarrow A^{\frac{1}{2}} \nabla y \quad \text { in } L^{p}(\Omega)^{N} .
$$

Thus, to complete the proof it remains to note that

$$
\int_{\Omega}\left|\left(\nabla \varphi, A_{k} \nabla \varphi\right)\right|^{\frac{p-2}{2}}\left(\nabla y_{k}, A_{k} \nabla \varphi\right) d x=\int_{\Omega}\left(\left|A_{k}^{\frac{1}{2}} \nabla \varphi\right|^{p-2} A_{k}^{\frac{1}{2}} \nabla \varphi, A_{k}^{\frac{1}{2}} \nabla y_{k}\right) d x
$$

and apply properties (3.3) and (3.5).

As an obvious consequence of this result, we have the following property.

Corollary 3.3. Let $\left\{\left(A_{k}, y_{k}\right) \in \Xi\right\}_{k \in \mathbb{N}}$ and $\left\{\zeta_{k} \in W_{0}^{1, q}(\Omega)\right\}_{k \in \mathbb{N}}$ be sequences such that $A_{k}^{\frac{1}{2}} \stackrel{*}{\rightarrow} A^{\frac{1}{2}}$ in $B V\left(\Omega ; \mathbb{S}^{N}\right), y_{k} \rightarrow y$ in $W_{0}^{1, p}(\Omega)$, and $\zeta_{k} \rightarrow \zeta$ in $W_{0}^{1, q}(\Omega)$. Then

$$
\begin{aligned}
& \lim _{k \rightarrow \infty} \int_{\Omega}\left|\left(\nabla \zeta_{k}, A_{k} \nabla \zeta_{k}\right)\right|^{\frac{p-2}{2}}\left(\nabla y_{k}, A_{k} \nabla \zeta_{k}\right) d x \\
& \quad=\int_{\Omega}|(\nabla \zeta, A \nabla \zeta)|^{\frac{p-2}{2}}(\nabla y, A \nabla \zeta) d x .
\end{aligned}
$$

Our next step concerns the study of topological properties of the set of admissible solutions $\Xi$ to problem (1.1).

The following result is crucial for our further analysis.

Theorem 3.4. Let $\left\{\left(A_{k}, y_{k}\right)\right\}_{k \in \mathbb{N}} \subset \Xi$ be an arbitrary sequence. Then there is a pair $(A, y) \in \Xi$ such that, up to a subsequence, $A_{k}^{\frac{1}{2}} \stackrel{*}{\rightarrow} A^{\frac{1}{2}}$ in $B V\left(\Omega ; \mathbb{S}^{N}\right)$ and $y_{k} \rightarrow y$ in $W_{0}^{1, p}(\Omega)$. 
Proof. As follows from definition of the set $\Xi$ (see (2.3)) and a priori estimate (2.14) the sequence $\left\{\left(A_{k}^{\frac{1}{2}}, y_{k}\right)\right\}_{k \in \mathbb{N}}$ is bounded in $B V\left(\Omega ; \mathbb{S}^{N}\right) \times W_{0}^{1, p}(\Omega)$.

By Remark 3.1 and the compactness properties of $B V\left(\Omega ; \mathbb{S}^{N}\right) \times W_{0}^{1, p}(\Omega)$, there exists a subsequence of $\left\{\left(A_{k}, y_{k}\right) \in \Xi\right\}_{k \in \mathbb{N}}$, still denoted by the same indices, and a symmetric matrix $A$ and a distribution $y \in W_{0}^{1, p}(\Omega)$ such that

$$
\begin{aligned}
& A_{k}^{\frac{1}{2}} \rightarrow A^{\frac{1}{2}} \text { in } L^{1}\left(\Omega ; \mathbb{S}^{N}\right), \quad y_{k} \rightarrow y \text { in } W_{0}^{1, p}(\Omega), \\
& A^{\frac{1}{2}} \in B V\left(\Omega ; \mathbb{S}^{N}\right), \quad A_{k}^{\frac{1}{2}} \rightarrow A^{\frac{1}{2}} \text { almost everywhere in } \Omega, \\
& \int_{\Omega}\left|D A^{\frac{1}{2}}\right| \leq \liminf _{k \rightarrow \infty} \int_{\Omega}\left|D A_{k}^{\frac{1}{2}}\right| \leq \gamma .
\end{aligned}
$$

Since $y_{k} \in K$ for all $k \in \mathbb{N}$ and the set $K$ is a closed convex subset of $W_{0}^{1, p}(\Omega)$, by Mazur's lemma this set is closed with respect to the weak topology of $W_{0}^{1, p}(\Omega)$. Hence, $y \in K$. Moreover, as follows from (3.7) and definition of the set $\mathfrak{A}_{a d}$, the inequality

$$
\xi_{1}^{2}\|\eta\|^{2} \leq(\eta, A \eta) \leq \xi_{2}^{2}\|\eta\|^{2} \quad \text { a.e. in } \Omega \forall \eta \in \mathbb{R}^{N},
$$

is valid. Thus, $A \in \mathfrak{A}_{a d}$. To complete the proof it is enough to show that the limit pair $(A, y)$ is related by variational inequality $(2.13)$. With that in mind we write down this inequality for $\left(A_{k}, y_{k}\right)$ :

$$
\int_{\Omega}\left|\left(A_{k} \nabla v, \nabla v\right)\right|^{\frac{p-2}{2}}\left(A_{k} \nabla v, \nabla v-\nabla y_{k}\right) d x \geq \int_{\Omega} f\left(v-y_{k}\right) d x, \quad \forall v \in K,
$$

and pass to the limit in it as $k \rightarrow \infty$.

In view of properties (3.6)-(3.9) and boundedness of $\left\{A_{k}\right\}_{k \in \mathbb{N}}$ in $L^{\infty}\left(\Omega ; \mathbb{S}^{N}\right)$, by Lebesgue's Theorem we get that $A_{k}^{\frac{1}{2}} \nabla \varphi \rightarrow A^{\frac{1}{2}} \nabla \varphi$ strongly in $L^{p}(\Omega)^{N}$ for every $\varphi \in C_{0}^{\infty}(\Omega)$. Therefore,

$$
\begin{aligned}
& \lim _{k \rightarrow \infty} \int_{\Omega}\left|\left(A_{k} \nabla v, \nabla v\right)\right|^{\frac{p-2}{2}}\left(A_{k} \nabla v, \nabla v\right) d x \\
& =\lim _{k \rightarrow \infty} \int_{\Omega}\left|A_{k}^{\frac{1}{2}} \nabla v\right|^{p-2}\left(A_{k}^{\frac{1}{2}} \nabla v, A_{k}^{\frac{1}{2}} \nabla v\right) d x=\lim _{k \rightarrow \infty} \int_{\Omega}\left|A_{k}^{\frac{1}{2}} \nabla v\right|^{p} d x \\
& =\int_{\Omega}\left|A^{\frac{1}{2}} \nabla v\right|^{p-2}(\nabla v, A \nabla v) d x=\int_{\Omega}|(A \nabla v, \nabla v)|^{\frac{p-2}{2}}(A \nabla v, \nabla v) d x
\end{aligned}
$$

and

$$
\begin{aligned}
\lim _{k \rightarrow \infty} \int_{\Omega}\left|\left(A_{k} \nabla v, \nabla v\right)\right|^{\frac{p-2}{2}}\left(A_{k} \nabla v, \nabla y_{k}\right) d x \\
\stackrel{\text { by Lemma } 3.2}{=} \int_{\Omega}|(A \nabla v, \nabla v)|^{\frac{p-2}{2}}(A \nabla v, \nabla y) d x .
\end{aligned}
$$

We, thus, can pass to the limit in relation (3.10) as $k \rightarrow \infty$ and arrive at the inequality (2.13), which means that $y \in K$ is a solution to variational problem (2.4). This fact together with $A \in \mathfrak{A}_{a d}$ leads us to the conclusion: $(A, y) \in \Xi$, i.e. the limit pair $(A, y)$ is admissible to optimal control problem (1.1). The proof is complete. 
In conclusion of this section, we give the existence result for optimal control problem (1.1) which is an immediate consequence of the compactness properties of $B V\left(\Omega ; \mathbb{S}^{N}\right)$, a priory estimate (2.14), Theorem 2.2, and compactness of embedding $W_{0}^{1, p}(\Omega) \hookrightarrow L^{2}(\Omega)$.

Theorem 3.5. Let $y_{d} \in L^{2}(\Omega)$ and $f \in L^{2}(\Omega)$ be given distributions. Then optimal control problem (1.1) admits at least one solution $\left(A^{\text {opt }}, y^{\text {opt }}\right) \in \Xi$.

\section{Regularization of OCP (1.1)}

As was pointed out in [17], the anisotropic $p$-Laplacian $\Delta_{p}(A, y)$ provides an example of a quasi-linear operator in divergence form with a so-called degenerate nonlinearity for $p>2$. In this context we have non-differentiability of the state $y$ with respect to the matrix-valued control $A$. As follows from Theorem 3.5, this fact is not an obstacle to prove existence of optimal controls in the coefficients, but it causes certain difficulties when deriving the optimality conditions for the considered problem. To overcome this difficulty, we introduce the following family of approximating control problems (see, for comparison, the approach of Casas and Fernandez [5] for quasi-linear elliptic variational inequalities with a distributed control in the right hand side)

$$
\text { Minimize } \quad I_{\varepsilon, k}(A, y)=\int_{\Omega}\left|y(x)-y_{d}(x)\right|^{2} d x
$$

subject to the constraints

$$
\begin{aligned}
\left\langle-\Delta_{\varepsilon, k, p}(A, y), v-y\right\rangle_{H^{-1}(\Omega) ; H_{0}^{1}(\Omega)} & \geq\langle f, v-y\rangle_{H^{-1}(\Omega) ; H_{0}^{1}(\Omega)}, \quad \forall v \in K^{*}, \\
y & \in K^{*} \\
A & \in \mathfrak{A}_{a d} .
\end{aligned}
$$

Here,

$$
\mathfrak{A}_{a d}=\left\{\begin{array}{ll}
A \in L^{1}\left(\Omega ; \mathbb{S}^{N}\right) & \begin{array}{l}
\xi_{1}^{2}\|\eta\|^{2} \leq(\eta, A \eta) \leq \xi_{2}^{2}\|\eta\|^{2} \text { a.e. in } \Omega \forall \eta \in \mathbb{R}^{N}, \\
A^{\frac{1}{2}} \in B V\left(\Omega ; \mathbb{S}^{N}\right), \quad \int_{\Omega}\left|D A^{\frac{1}{2}}\right| \leq \gamma
\end{array}
\end{array}\right\}
$$

$k \in \mathbb{N}, \varepsilon$ is a small parameter, which varies within a strictly decreasing sequence of positive numbers converging to 0 , the set $K^{*}$ is defined in Remark 2.4, and

$$
\Delta_{\varepsilon, k, p}(A, y)=\operatorname{div}\left(\left[\varepsilon+\mathcal{F}_{k}\left(\left|A^{\frac{1}{2}} \nabla y\right|^{2}\right)\right]^{\frac{p-2}{2}} A(x) \nabla y\right),
$$

where $\mathcal{F}_{k}: \mathbb{R}_{+} \rightarrow \mathbb{R}_{+}$is a non-decreasing $C^{1}\left(\mathbb{R}_{+}\right)$-function such that

$$
\begin{array}{r}
\mathcal{F}_{k}(t)=t, \quad \text { if } t \in\left[0, k^{2}\right], \quad \mathcal{F}_{k}(t)=k^{2}+1, \quad \text { if } t>k^{2}+1, \quad \text { and } \\
t \leq \mathcal{F}_{k}(t) \leq t+\delta, \quad \text { if } \quad k^{2} \leq t<k^{2}+1 \quad \text { for some } \delta \in(0,1) .
\end{array}
$$

The main goal of this section is to show that, for each $\varepsilon>0$ and $k \in \mathbb{N}$, the approximating optimal control problem (4.1)-(4.4) is well posed and its solutions are uniformly bounded in appropriated Banach spaces with respect to parameters $\varepsilon>0$ and $k \in \mathbb{N}$. 
Remark 4.1. Since $K^{*}$ is a closed convex and bounded subset of $W_{0}^{1, p}(\Omega)$, and $W_{0}^{1, p}(\Omega)$ is continuously embedded to $H_{0}^{1}(\Omega)$, it follows that $K^{*}$ is the closed convex subset of $H_{0}^{1}(\Omega)$.

Remark 4.2. We note that the main effect of the perturbations of anisotropic $p$ Laplacian $\Delta_{p}(A, y)$ in the form $\Delta_{\varepsilon, k, p}(A, y)$ is its regularization around critical points and points where the function $\left|A^{\frac{1}{2}} \nabla y\right|$ becomes unbounded. In particular, if $y \in W_{0}^{1, p}(\Omega), A \in \mathfrak{A}_{a d}, \Omega_{k}(A, y):=\left\{x \in \Omega:\left|A^{\frac{1}{2}} \nabla y\right|>\sqrt{k^{2}+1}\right\}$, then the following chain of inequalities

$$
\begin{aligned}
& \left|\Omega_{k}(A, y)\right|:=\int_{\Omega_{k}(A, y)} 1 d x \leq \frac{1}{\sqrt{k^{2}+1}} \int_{\Omega_{k}(A, y)}\left|A^{\frac{1}{2}} \nabla y\right| d x \\
& \quad \leq \frac{\left|\Omega_{k}(A, y)\right|^{\frac{1}{q}}}{\sqrt{k^{2}+1}}\left(\int_{\Omega}\left|A^{\frac{1}{2}} \nabla y\right|^{p} d x\right)^{\frac{1}{p}} \leq \frac{\left\|\xi_{2}\right\|_{L^{\infty}(\Omega)}\|y\|_{W_{0}^{1, p}(\Omega)}}{\sqrt{k^{2}+1}}\left|\Omega_{k}(A, y)\right|^{\frac{p-1}{p}}
\end{aligned}
$$

shows that the Lebesgue measure of the set $\Omega_{k}(A, y)$ satisfies the estimate

$$
\begin{aligned}
& \left|\Omega_{k}(A, y)\right| \leq\left(\frac{\left\|\xi_{2}\right\|_{L^{\infty}(\Omega)}}{\sqrt{k^{2}+1}}\right)^{p}\|y\|_{W_{0}^{1, p}(\Omega)}^{p} \\
& \leq \frac{\|y\|_{W_{0}^{1, p}(\Omega)}^{p}\left\|\xi_{2}\right\|_{L^{\infty}(\Omega)}^{p}}{k^{p}}, \quad \forall y \in W_{0}^{1, p}(\Omega),
\end{aligned}
$$

i.e. the approximation $\mathcal{F}_{k}\left(\left|A^{\frac{1}{2}} \nabla y\right|^{2}\right)$ is essential on sets with small Lebesgue measure. At the same time, if instead of element $y \in W_{0}^{1, p}(\Omega)$ we consider an element $y^{*} \in H_{0}^{1}(\Omega)$, then its level set

$$
\Omega_{k}\left(A, y^{*}\right):=\left\{x \in \Omega:\left|A^{\frac{1}{2}} \nabla y^{*}\right|>\sqrt{k^{2}+1}\right\}
$$

may have other than (4.6) characteristics. Indeed,

$$
\begin{aligned}
\left|\Omega_{k}\left(A, y^{*}\right)\right| & :=\int_{\Omega_{k}\left(A, y^{*}\right)} 1 d x \leq \frac{1}{\sqrt{k^{2}+1}} \int_{\Omega_{k}\left(A, y^{*}\right)}\left|A^{\frac{1}{2}} \nabla y^{*}\right| d x \\
& \leq \frac{\left|\Omega_{k}\left(A, y^{*}\right)\right|^{\frac{1}{2}}}{k} \times\left(\int_{\Omega_{k}\left(A, y^{*}\right)}\left|A^{\frac{1}{2}} \nabla y^{*}\right|^{2} d x\right)^{\frac{1}{2}} \\
& \leq \frac{\left\|\xi_{2}\right\|_{L^{\infty}(\Omega)}}{k}\left|\Omega_{k}\left(A, y^{*}\right)\right|^{\frac{1}{2}}\left\|y^{*}\right\|_{H_{0}^{1}(\Omega)} .
\end{aligned}
$$

Hence, the Lebesgue measure of the set $\Omega_{k}\left(A, y^{*}\right)$ satisfies the estimate

$$
\left|\Omega_{k}\left(A, y^{*}\right)\right| \leq \frac{\left\|\xi_{2}\right\|_{L^{\infty}(\Omega)}^{2}}{k^{2}}\left\|y^{*}\right\|_{H_{0}^{1}(\Omega)}^{2} .
$$

We begin with a few auxiliary results concerning monotonicity and growth conditions for the regularized anisotropic $p$-Laplacian $\Delta_{\varepsilon, k, p}(A, \cdot)$.

Proposition 4.3. For every $A \in \mathfrak{A}_{a d}, k \in \mathbb{N}$, and $\varepsilon>0$, the operator $\mathcal{A}_{\varepsilon, k}:=$ $-\Delta_{\varepsilon, k, p}(A, \cdot): H_{0}^{1}(\Omega) \rightarrow H^{-1}(\Omega)$ is bounded. 
Proof. From the assumptions on $\mathcal{F}_{k}$ and boundedness of $A$, we get

$$
\begin{aligned}
\left\|\mathcal{A}_{\varepsilon, k}\right\| & =\sup _{\|y\|_{H_{0}^{1}(\Omega)} \leq 1}\left\|\mathcal{A}_{\varepsilon, k} y\right\|_{H^{-1}(\Omega)}=\sup _{\|y\|_{H_{0}^{1}(\Omega)} \leq 1\|v\|_{H_{0}^{1}(\Omega)} \leq 1} \sup _{\left.\| \mathcal{A}_{\varepsilon, k} y, v\right\rangle \mid} \sup _{\|y\|_{H_{0}^{1}(\Omega)} \leq 1\|v\|_{H_{0}^{1}(\Omega)} \leq 1} \int_{\Omega}\left[\varepsilon+\mathcal{F}_{k}\left(\left|A^{\frac{1}{2}} \nabla y\right|^{2}\right)\right]^{\frac{p-2}{2}}|(\nabla v, A \nabla y)| d x \\
& \leq \frac{\left\|\xi_{2}\right\|_{L^{\infty}(\Omega)}^{2}}{\left(\varepsilon+k^{2}+1\right)^{\frac{2-p}{2}}} \sup _{\|y\|_{H_{0}^{1}(\Omega)} \leq 1\|v\|_{H_{0}^{1}(\Omega)} \leq 1} \sup _{\|y\|_{H_{0}^{1}(\Omega)}\|v\|_{H_{0}^{1}(\Omega)}=C_{\varepsilon, k},}
\end{aligned}
$$

which concludes the proof.

Proposition 4.4. For every $A \in \mathfrak{A}_{a d}, k \in \mathbb{N}$, and $\varepsilon>0$, the operator $\mathcal{A}_{\varepsilon, k}$ is strictly monotone.

Proof. To begin with, we make use of the following algebraic inequality:

$$
\begin{aligned}
& \left(\left(\varepsilon+\mathcal{F}_{k}\left(|a|^{2}\right)\right)^{\frac{p-2}{2}} a-\left(\varepsilon+\mathcal{F}_{k}\left(|b|^{2}\right)\right)^{\frac{p-2}{2}} b, a-b\right) \\
& \geq \varepsilon^{\frac{p-2}{2}}|a-b|^{2}, \forall a, b \in \mathbb{R}^{N} .
\end{aligned}
$$

In order to prove it, we note that the left hand side of (4.8) can be rewritten as follows

$$
\begin{aligned}
( & \left.\left(\varepsilon+\mathcal{F}_{k}\left(|a|^{2}\right)\right)^{\frac{p-2}{2}} a-\left(\varepsilon+\mathcal{F}_{k}\left(|b|^{2}\right)\right)^{\frac{p-2}{2}} b, a-b\right) \\
= & \left(\int_{0}^{1} \frac{d}{d s}\left\{\left(\varepsilon+\mathcal{F}_{k}\left(|s a+(1-s) b|^{2}\right)\right)^{\frac{p-2}{2}}(s a+(1-s) b)\right\} d s, a-b\right) \\
= & \int_{0}^{1}\left(\varepsilon+\mathcal{F}_{k}\left(|s a+(1-s) b|^{2}\right)\right)^{\frac{p-2}{2}}|a-b|^{2} d s \\
& +(p-2) \int_{0}^{1}\left\{\left(\varepsilon+\mathcal{F}_{k}\left(|s a+(1-s) b|^{2}\right)\right)^{\frac{p-4}{2}} \mathcal{F}_{k}^{\prime}\left(|s a+(1-s) b|^{2}\right)\right. \\
& \left.\times|(s a+(1-s) b, a-b)|^{2}\right\} d s=I_{1}+I_{2} .
\end{aligned}
$$

Since $p \geq 2$ and $\mathcal{F}_{k}: \mathbb{R}_{+} \rightarrow \mathbb{R}_{+}$is a non-decreasing $C^{1}\left(\mathbb{R}_{+}\right)$-function, it follows that $I_{2} \geq 0$ for all $a, b \in \mathbb{R}^{N}$. It remains to observe that $\left(\varepsilon+\mathcal{F}_{k}(\mid s a+\right.$ $\left.\left.\left.(1-s) b\right|^{2}\right)\right) \geq \varepsilon, \forall a, b \in \mathbb{R}^{N}$. Hence, $I_{1} \geq \varepsilon^{\frac{p-2}{2}}|a-b|^{2}$ and we arrive at the inequality (4.8). With this we obtain

$$
\begin{aligned}
&\left\langle-\Delta_{\varepsilon, k, p}(A, y)+\Delta_{\varepsilon, k, p}(A, v), y-v\right\rangle_{H^{-1}(\Omega) ; H_{0}^{1}(\Omega)} \int_{\Omega}\left(\left(\varepsilon+\mathcal{F}_{k}\left(\left|A^{\frac{1}{2}} \nabla y\right|^{2}\right)\right)^{\frac{p-2}{2}} \nabla y, A(\nabla y-\nabla v)\right)_{\mathbb{R}^{N}} d x \\
&- \int_{\Omega}\left(\left(\varepsilon+\mathcal{F}_{k}\left(\left|A^{\frac{1}{2}} \nabla v\right|^{2}\right)\right)^{\frac{p-2}{2}} \nabla v, A(\nabla y-\nabla v)\right)_{\mathbb{R}^{N}} d x \\
& \text { having put }\left\{a:=A^{\frac{1}{2}} \nabla y, \quad b:=A^{\frac{1}{2}} \nabla v\right\}, \text { we get }
\end{aligned}
$$




$$
\begin{aligned}
& =\int_{\Omega}\left(\left(\varepsilon+\mathcal{F}_{k}\left(|a|^{2}\right)\right)^{\frac{p-2}{2}} a-\left(\varepsilon+\mathcal{F}_{k}\left(|b|^{2}\right)\right)^{\frac{p-2}{2}} b, a-b\right) d x \\
& \geq \varepsilon^{\frac{p-2}{2}} \int_{\Omega}\left|A^{\frac{1}{2}} \nabla y-A^{\frac{1}{2}} \nabla v\right|^{2} d x=\varepsilon^{\frac{p-2}{2}} \int_{\Omega}(\nabla y-\nabla v, A(\nabla y-\nabla v)) d x \\
& \geq \alpha^{2} \varepsilon^{\frac{p-2}{2}}\|y-v\|_{H_{0}^{1}(\Omega)}^{2} \geq 0 .
\end{aligned}
$$

Since the relation

$$
\left\langle-\Delta_{\varepsilon, k, p}(A, y)+\Delta_{\varepsilon, k, p}(A, v), y-v\right\rangle_{H^{-1}(\Omega) ; H_{0}^{1}(\Omega)}=0
$$

implies that $\nabla y=\nabla v$ a. e. in $\Omega$, it follows that the strict monotonicity property (2.10) holds true for each $A \in \mathfrak{A}_{a d}, k \in \mathbb{N}$, and $\varepsilon>0$.

Proposition 4.5. For every $A \in \mathfrak{A}_{a d}, k \in \mathbb{N}$, and $\varepsilon>0$, the operator $\mathcal{A}_{\varepsilon, k}$ is coercive (in the sense of relation (2.6)).

Proof. The coercivity property obviously follows from (2.6) under $v_{0}=0 \in K^{*}$ and the estimate

$$
\left\langle-\Delta_{\varepsilon, k, p}(A, y), y\right\rangle_{H^{-1}(\Omega) ; H_{0}^{1}(\Omega)} \geq \alpha^{2} \varepsilon^{\frac{p-2}{2}}\|y\|_{H_{0}^{1}(\Omega)}^{2} .
$$

Proposition 4.6. For every $A \in \mathfrak{A}_{a d}, k \in \mathbb{N}$, and $\varepsilon>0$, the operator $\mathcal{A}_{\varepsilon, k}$ is semicontinuous.

Proof. Indeed, in order to get the equality

$$
\lim _{t \rightarrow 0}\left\langle-\Delta_{\varepsilon, k, p}(A, y+t w), v\right\rangle_{H^{-1}(\Omega) ; H_{0}^{1}(\Omega)}=\left\langle-\Delta_{\varepsilon, k, p}(A, y), v\right\rangle_{H^{-1}(\Omega) ; H_{0}^{1}(\Omega)},
$$

it is enough to observe that

$\left(\varepsilon+\mathcal{F}_{k}\left(\left|A^{\frac{1}{2}}(\nabla y+t \nabla w)\right|^{2}\right)\right)^{\frac{p-2}{2}} A(\nabla y+t \nabla w) \rightarrow\left(\varepsilon+\mathcal{F}_{k}\left(\left|A^{\frac{1}{2}} \nabla y\right|^{2}\right)\right)^{\frac{p-2}{2}} A \nabla y$

as $t \rightarrow 0$ almost everywhere in $\Omega$, and make use of Lebesgue's dominated convergence theorem.

Taking into account the fact that the set $K^{*}$, defined in Remark 2.4, is a nonempty convex closed subset of $H_{0}^{1}(\Omega)$, we can apply the abstract theorem on monotone operators (see Theorem 2.2) to the variational inequality

$$
\begin{aligned}
& \text { Find } y \in K^{*} \text { s.t. }\left\langle\mathcal{A}_{\varepsilon, k} y, v-y\right\rangle_{H^{-1}(\Omega) ; H_{0}^{1}(\Omega)} \\
& \geq\langle f, v-y\rangle_{H^{-1}(\Omega) ; H_{0}^{1}(\Omega)}, \quad \forall v \in K^{*} .
\end{aligned}
$$

As a result, closely following the arguments of Sect. 2 , we arrive at the following assertion.

Theorem 4.7. For each $\varepsilon>0, k \in \mathbb{N}, A \in \mathfrak{A}_{a d}$, and $f \in L^{2}(\Omega)$, the variational inequality (4.2)-(4.3) admits a unique solution $y_{\varepsilon, k} \in K^{*} \subset H_{0}^{1}(\Omega)$ such that

$$
\begin{gathered}
\int_{\Omega}\left(\varepsilon+\mathcal{F}_{k}\left(\left|A^{\frac{1}{2}} \nabla v\right|^{2}\right)\right)^{\frac{p-2}{2}}\left(A \nabla v, \nabla v-\nabla y_{\varepsilon, k}\right) d x \\
\geq \int_{\Omega} f\left(v-y_{\varepsilon, k}\right) d x, \quad \forall v \in K^{*} .
\end{gathered}
$$


Remark 4.8. It is easy to show, that the set $\left\{y_{\varepsilon, k} \in K^{*} \mid A \in \mathfrak{A}_{a d},\left(A, y_{\varepsilon, k}\right)\right.$ are related by inequality $\left.(4.11)\right\} \subset H_{0}^{1}(\Omega)$ is bounded in $H_{0}^{1}(\Omega)$-norm for every $k \in \mathbb{N}$ and $\varepsilon>0$. Indeed, since by the initial assumptions the set $K^{*}$ contains zero element of $H_{0}^{1}(\Omega)$, it follows that

$$
\begin{aligned}
& \varepsilon^{\frac{p-2}{2}} \alpha^{2}\left\|y_{\varepsilon, k}\right\|_{H_{0}^{1}(\Omega)}^{2} \stackrel{\text { by }(4.9)}{\leq}\left\langle\mathcal{A}_{\varepsilon, k} y_{\varepsilon, k}, y_{\varepsilon, k}\right\rangle_{H^{-1}(\Omega) ; H_{0}^{1}(\Omega)} \\
& \text { by }(4.10) \\
& \stackrel{\text { s. }}{\leq}\left\langle f, y_{\varepsilon, k}\right\rangle_{H^{-1}(\Omega) ; H_{0}^{1}(\Omega)} \leq\|f\|_{L^{2}(\Omega)}\left\|y_{\varepsilon, k}\right\|_{H_{0}^{1}(\Omega)} .
\end{aligned}
$$

Hence, $\sup _{A \in \mathfrak{A}_{a d}}\left\|y_{\varepsilon, k}\right\|_{H_{0}^{1}(\Omega)} \leq \varepsilon^{\frac{2-p}{2}} \alpha^{-2}\|f\|_{L^{2}(\Omega)}$.

Thus, as follows from Theorem 4.7, for every $\varepsilon>0$ and $k \in \mathbb{N}$, the set of admissible pairs to the approximating optimal control problem (4.1)-(4.4)

$$
\Xi_{\varepsilon, k}=\left\{(A, y) \mid A \in \mathfrak{A}_{a d}, y \in K^{*} \subset H_{0}^{1}(\Omega),(A, y) \text { are related by }(4.11)\right\}
$$

is nonempty. Moreover, for every sequence $\left\{\left(A_{n}, y_{n}\right) \in \Xi_{\varepsilon, k}\right\}_{n \in \mathbb{N}}$, in view of Remark 4.8, we have

$$
\sup _{n \in \mathbb{N}}\left[\left\|A_{n}^{\frac{1}{2}}\right\|_{B V\left(\Omega ; \mathbb{S}^{N}\right)}+\left\|y_{n}\right\|_{H_{0}^{1}(\Omega)}\right] \leq \sqrt{N}\left\|\xi_{2}\right\|_{L^{1}(\Omega)}+\gamma+\varepsilon^{\frac{2-p}{2}} \alpha^{-2}\|f\|_{L^{2}(\Omega)} .
$$

Hence (see Remark 3.1), there exists a subsequence $\left\{\left(A_{n_{i}}, y_{n_{i}}\right)\right\}_{i \in \mathbb{N}}$ and a pair $(A, y) \in B V(\Omega) \times H_{0}^{1}(\Omega)$ such that

$$
\begin{aligned}
& y_{n_{i}} \rightarrow y \text { in } H_{0}^{1}(\Omega), \quad y_{n_{i}} \rightarrow y \text { in } L^{2}(\Omega), \\
& A_{n_{i}}^{\frac{1}{2}} \rightarrow A^{\frac{1}{2}} \quad \text { in } L^{1}(\Omega), \quad A_{n_{i}}^{\frac{1}{2}} \rightarrow A^{\frac{1}{2}} \quad \text { almost everywhere in } \Omega, \\
& \gamma \geq \liminf _{i \rightarrow \infty} \int_{\Omega}\left|D A_{n_{i}}^{\frac{1}{2}}\right| \geq \int_{\Omega}\left|D A^{\frac{1}{2}}\right| .
\end{aligned}
$$

By analogy with Theorem 3.4, it is easy to show that the set $\Xi_{\varepsilon, k}$ is sequentially closed with respect to the convergence (4.13) in $B V(\Omega) \times H_{0}^{1}(\Omega)$. Therefore, $(A, y) \in \Xi_{\varepsilon, k}$. Moreover, since $\left\{y_{n_{i}}\right\}_{i \in \mathbb{N}} \subset K^{*}$ and $K^{*}$ is a closed convex subset of $H_{0}^{1}(\Omega)$, then the weak convergence $y_{n_{i}} \rightarrow y$ in $H_{0}^{1}(\Omega)$ implies both the inclusion $y \in K^{*}$ and the strong convergence $y_{i} \rightarrow y$ in $L^{2}(\Omega)$ by RellichKondrachov compactness theorem. As a result, we have

$$
\liminf _{i \rightarrow \infty} I_{\varepsilon, k}\left(A_{n_{i}}, y_{n_{i}}\right)=I_{\varepsilon, k}(A, y)
$$

whenever $\left\{\left(A_{n_{i}}, y_{n_{i}}\right)\right\}_{i \in \mathbb{N}} \subset \Xi_{\varepsilon, k}$ converges to $(A, y)$ in the sense of (4.13).

Thus, since the cost functional (4.1) is bounded below on $\Xi_{\varepsilon, k}$ and $I_{\varepsilon, k}(A, y)<$ $+\infty$ for all $(A, y) \in \Xi_{\varepsilon, k}$, the direct method of Calculus of Variations immediately leads us to the following conclusion.

Theorem 4.9. For every positive value $\varepsilon>0$ and integer $k \in \mathbb{N}$, the optimal control problem (4.1)-(4.4) is solvable, i.e. there exists at least one pair $\left(A_{\varepsilon, k}^{o p t}, y_{\varepsilon, k}^{o p t}\right) \in \Xi_{\varepsilon, k}$ such that

$$
I_{\varepsilon, k}\left(A_{\varepsilon, k}^{o p t}, y_{\varepsilon, k}^{o p t}\right)=\inf _{(A, y) \in \Xi_{\varepsilon, k}} I_{\varepsilon, k}(A, y)
$$




\section{Asymptotic analysis of the approximating OCP (4.1)-(4.4)}

Our main intention in this section is to show that some optimal solutions to original OCP (1.1) can be attained (in some sense) by optimal solutions to approximating problems (4.1)-(4.4). With that in mind, we make use of the concept of variational convergence of constrained minimization problems (see [11]). In order to study the asymptotic behaviour of a family of OCPs (4.1)(4.4), the passage to the limit in relations (4.1)-(4.4) as $\varepsilon \rightarrow 0$ and $k \rightarrow \infty$ has to be realized. The expression "passing to the limit" means that we have to find a kind of "limit cost functional" $I$ and "limit set of constraints" $\Xi$ with a

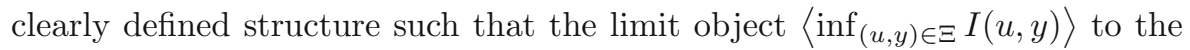
family (4.1)-(4.4) could be interpreted as some OCP.

We are now in a position to show that optimal pairs to approximating OCP (4.1)-(4.4) lead in the limit to some optimal solution to original OCP (1.1). To begin with, we state the following result.

Theorem 5.1. Let $y_{d} \in L^{2}(\Omega), f \in L^{2}(\Omega)$, and $\xi_{1}, \xi_{2} \in L^{\infty}(\Omega) \cap B V(\Omega)$ be arbitrary distributions with property (2.2). Then, for a given $p \geq 2$, the sequence of sets $\left\{\Xi_{\varepsilon, k}\right\}_{\substack{\varepsilon>0 \\ k \in \mathbb{N}}} \subset B V(\Omega) \times H_{0}^{1}(\Omega)$ converges to the set $\Xi \overline{a s} \varepsilon \rightarrow 0$ and $k \rightarrow \infty$ in Kuratowski sense as follows:

(d) If sequences $\left\{\varepsilon_{n}\right\}_{n \in \mathbb{N}},\left\{k_{n}\right\}_{n \in \mathbb{N}}$, and $\left\{\left(A_{n}, y_{n}\right)\right\}_{n \in \mathbb{N}}$ are such that $\varepsilon_{n} \rightarrow 0$ and $k_{n} \rightarrow \infty$ as $n \rightarrow \infty,\left(A_{n}, y_{n}\right) \in \Xi_{\varepsilon_{n}, k_{n}}, \forall n \in \mathbb{N}, A_{n}^{\frac{1}{2}} \stackrel{*}{\rightarrow} A^{\frac{1}{2}}$ in $B V\left(\Omega ; \mathbb{S}^{N}\right)$, and $y_{n} \rightarrow y$ in $H_{0}^{1}(\Omega)$, then

$$
(A, y) \in \Xi \text {; }
$$

(dd) For every $(A, y) \in \Xi$, there exists a sequence $\left\{\left(A_{\varepsilon, k}, y_{\varepsilon, k}\right)\right\}_{\substack{\varepsilon>0 \\ k \in \mathbb{N}}}$ such that

$$
\begin{aligned}
& \left(A_{\varepsilon, k}, y_{\varepsilon, k}\right) \in \Xi_{\varepsilon, k}, \quad \forall \varepsilon>0, \forall k \in \mathbb{N}, \\
& y_{\varepsilon, k} \rightarrow y \quad \text { in } H_{0}^{1}(\Omega), \quad A_{\varepsilon, k}^{\frac{1}{2}} \stackrel{*}{\rightarrow} A^{\frac{1}{2}} \quad \text { in } B V\left(\Omega ; \mathbb{S}^{N}\right) .
\end{aligned}
$$

Proof. We begin with property (d). Let $\left\{\varepsilon_{n}\right\}_{n \in \mathbb{N}},\left\{k_{n}\right\}_{n \in \mathbb{N}}$, and $\left\{\left(A_{n}, y_{n}\right)\right\}_{n \in \mathbb{N}}$ be sequences satisfying all assumptions of item $(\mathrm{d})$. It is easy to see that $y \in$ $K^{*} \subset K \subset W_{0}^{1, p}(\Omega)$ due to closedness of $K^{*}$ with respect to weak convergence in $H_{0}^{1}(\Omega)$, and $A$ is an admissible control $\left(A \in \mathfrak{A}_{a d}\right)$. It remains to show that the limit pair $(A, y)$ is related by inequality (2.13). In fact, according to Remark 2.4 it is enough to show, that the limit pair $(A, y)$ is related by inequality (2.15). To this end, let us fix an arbitrary distribution $v \in K^{*}$ and pass to the limit in the relation (see Theorem 4.7)

$$
\int_{\Omega}\left(\varepsilon_{n}+\mathcal{F}_{k_{n}}\left(\left|A_{n}^{\frac{1}{2}} \nabla v\right|^{2}\right)\right)^{\frac{p-2}{2}}\left(A_{n} \nabla v, \nabla v-\nabla y_{n}\right) d x \geq \int_{\Omega} f\left(v-y_{n}\right) d x
$$

as $n \rightarrow \infty$. Taking into account Lebesgue Theorem and the facts that $\mathcal{F}_{k_{n}}(t) \rightarrow t \quad$ everywhere in $\mathbb{R}_{+}^{1}, A, A_{n} \in L^{\infty}\left(\Omega ; \mathbb{S}^{N}\right)$, $A_{n}^{\frac{1}{2}} \rightarrow A^{\frac{1}{2}} \quad$ almost everywhere in $\Omega$, $\left(\varepsilon_{n}+\mathcal{F}_{k_{n}}\left(|\nabla v|^{2}\right)\right)^{\frac{p-2}{2}} \nabla v \rightarrow|\nabla v|^{p-2} \nabla v \quad$ strongly in $L^{q}(\Omega)^{N}$, 
and making use of Lemma 3.2 and its Corollary 3.3, we get

$$
\begin{aligned}
& \lim _{n \rightarrow \infty} \int_{\Omega}\left(\varepsilon_{n}+\mathcal{F}_{k_{n}}\left(\left|A_{n}^{\frac{1}{2}} \nabla v\right|^{2}\right)\right)^{\frac{p-2}{2}}\left(A_{n} \nabla v, \nabla v\right) d x \\
& \quad=\int_{\Omega}\left|A^{\frac{1}{2}} \nabla v\right|^{p-2}(A \nabla v, \nabla v) d x, \\
& \lim _{n \rightarrow \infty} \int_{\Omega}\left(\varepsilon_{n}+\mathcal{F}_{k_{n}}\left(\left|A_{n}^{\frac{1}{2}} \nabla v\right|^{2}\right)\right)^{\frac{p-2}{2}}\left(A_{n} \nabla v, \nabla y_{n}\right) d x \\
& \quad=\int_{\Omega}\left|A^{\frac{1}{2}} \nabla v\right|^{p-2}(A \nabla v, \nabla y) d x, \\
& \lim _{n \rightarrow \infty} \int_{\Omega} f\left(v-y_{n}\right) d x=\int_{\Omega} f(v-y) d x .
\end{aligned}
$$

Thus, upon passing to the limit in (5.4) as $n \rightarrow \infty$, we arrive at the following variational inequality

$$
\int_{\Omega}|(A \nabla v, \nabla v)|^{\frac{p-2}{2}}(A \nabla v, \nabla v-\nabla y) d x \geq \int_{\Omega} f(v-y) d x, \quad \forall v \in K^{*} .
$$

Since $y \in K^{*}$, it follows that $y=y(A)$ is a solution to variational problem (2.14), and, due to Remark 2.4, it is a solution to (2.13). Thus, $(A, y) \in \Xi$.

The next step is to prove property $(\mathrm{dd})$. Let $(A, y) \in \Xi$ be an arbitrary admissible pair to original OCP (1.1). We construct a sequence $\left\{\left(A_{\varepsilon, k}, y_{\varepsilon, k}\right)\right\}_{\substack{\varepsilon>0 \\ k \in \mathbb{N}}}$ as follows: $A_{\varepsilon, k} \equiv A$ for all $\varepsilon>0$ and $k \in \mathbb{N}$, and $y_{\varepsilon, k}$ is the corresponding solution to regularized variational inequality (4.2)-(4.3) under $A_{\varepsilon, k}=A$. Then, $\left(A_{\varepsilon, k}, y_{\varepsilon, k}\right) \in \Xi_{\varepsilon, k}$ for all $\varepsilon>0$ and $k \in \mathbb{N}$, and, as follows from Remark 4.8, the sequence $\left\{y_{\varepsilon, k}\right\}_{\substack{\varepsilon>0 \\ k \in \mathbb{N}}}$ is uniformly bounded in $H_{0}^{1}(\Omega)$ and, therefore, it is relatively compact with respect to the weak convergence in $H_{0}^{1}(\Omega)$. Hence, all cluster pairs $\left(A, y^{*}\right)$ of the sequence $\left\{\left(A_{\varepsilon, k}, y_{\varepsilon, k}\right)\right\}_{\substack{c>0 \\ k \in \mathbb{N}}}$ with respect to convergence $(5.3)$ in $B V\left(\Omega ; \mathbb{S}^{N}\right) \times H_{0}^{1}(\Omega)$ are such that $y^{*} \in K^{*} \subset W_{0}^{1, p}(\Omega)$. Moreover, reiterating the arguments of the previous step, it can be shown that each of the cluster pairs $\left(A, y^{*}\right)$ are related by inequality (2.13). Since variational problem (2.4) has a unique solution for each $A \in \mathfrak{A}_{a d}$, it means that pair $(A, y) \in \Xi$ is the limit to the whole sequence $\left\{\left(A_{\varepsilon, k}, y_{\varepsilon, k}\right)\right\} \begin{gathered}\substack{\varepsilon>0 \\ k \in \mathbb{N}} \\ \text {. }\end{gathered}$

The following result is central in this paper and it clarifies the approximating properties of perturbed optimal control problems (4.1)-(4.4).

Theorem 5.2. Let $\left\{\left(A_{\varepsilon, k}^{0}, y_{\varepsilon, k}^{0}\right) \in \Xi_{\varepsilon, k}\right\}_{\substack{\varepsilon>0 \\ k \in \mathbb{R}}}$ be an arbitrary sequence of optimal pairs to approximating problems (4.1)-(4.4). Then this sequence is relatively compact with respect to the convergence

$$
\begin{aligned}
& y_{\varepsilon, k}^{0} \rightarrow y^{0} \quad \text { in } H_{0}^{1}(\Omega), \quad\left(A_{\varepsilon, k}^{0}\right)^{\frac{1}{2}} \stackrel{*}{\rightarrow}\left(A^{0}\right)^{\frac{1}{2}} \quad \text { in } B V\left(\Omega ; \mathbb{S}^{N}\right), \\
& A_{\varepsilon, k}^{0} \rightarrow A^{0} \quad \text { in } L^{1}\left(\Omega ; \mathbb{S}^{N}\right)
\end{aligned}
$$


and each its cluster pair $\left(A^{0}, y^{0}\right)$ is such that $\left(A^{0}, y^{0}\right) \in \Xi$ and

$$
\begin{aligned}
& \inf _{(A, y) \in \Xi} I(A, y)=I\left(A^{0}, y^{0}\right)=\lim _{\substack{\varepsilon \rightarrow 0 \\
k \rightarrow \infty}} I_{\varepsilon, k}\left(A_{\varepsilon, k}^{0}, y_{\varepsilon, k}^{0}\right) \\
& \quad=\lim _{\substack{\varepsilon \rightarrow 0 \\
k \rightarrow \infty}} \inf _{(A, y) \in \Xi_{\varepsilon, k}} I_{\varepsilon, k}(A, y) .
\end{aligned}
$$

Proof. As definition of the class of admissible controls $\mathfrak{A}_{a d}$ and Remark 4.8 indicate, the sequence $\left\{\left(A_{\varepsilon, k}^{0}, y_{\varepsilon, k}^{0}\right) \in \Xi_{\varepsilon, k}\right\}_{\substack{\varepsilon>0 \\ k \in \mathbb{R}}}$ of optimal pairs is relatively compact with respect to convergence (5.6). To prove variational equality (5.7), it remains to apply the Rellich-Kondrachov compactness theorem and the following relation coming from properties (d)-(dd) of Theorem 5.1

$$
\begin{aligned}
& \lim _{i \rightarrow \infty} \inf _{(A, y) \in \Xi_{\varepsilon_{i}, k_{i}}} I_{\varepsilon_{i}, k_{i}}(A, y)=\lim _{i \rightarrow \infty} I_{\varepsilon_{i}, k_{i}}\left(A_{\varepsilon_{i}, k_{i}}^{0}, y_{\varepsilon_{i}, k_{i}}^{0}\right) \stackrel{\text { by }(\mathrm{d})}{=} I(\widehat{A}, \widehat{y}) \\
& \quad \geq \inf _{(A, y) \in \Xi} I(A, y)=I\left(A^{0}, y^{0}\right) \stackrel{\text { by }}{=}(\mathrm{dd}) \\
& \quad \geq \limsup _{\substack{\varepsilon \rightarrow 0 \\
k \rightarrow \infty}} I_{\varepsilon, k}\left(A^{0}, y_{\varepsilon, k}\right) \\
& \quad \operatorname{linf}_{\substack{\varepsilon \rightarrow \infty \\
k \rightarrow \infty}} I_{\varepsilon, k}(A, y) \geq \limsup _{i \rightarrow \infty} \inf _{(A, y) \in \Xi_{\varepsilon_{i}, k_{i}}} I_{\varepsilon_{i}, k_{i}}(A, y) \\
& \quad \lim _{i \rightarrow \infty} I_{\varepsilon_{i}, k_{i}}\left(A_{\varepsilon_{i}, k_{i}}^{0}, y_{\varepsilon_{i}, k_{i}}^{0}\right) .
\end{aligned}
$$

Remark 5.3. As follows from Theorems 5.2 and 5.1, whatever sequence of optimal solutions $\left\{\left(A_{\varepsilon, k}^{0}, y_{\varepsilon, k}^{0}\right)\right\}_{\substack{\varepsilon>0 \\ k \in \mathbb{N}}}$ to approximating problems (4.1)-(4.4) has been chosen, it always gives in the limit as $\varepsilon \rightarrow 0$ and $k \rightarrow \infty$ some optimal pair to original OCP (1.1). However, it is unknown whether any solution $\left(A^{0}, y^{0}\right)$ to OCP (1.1) can be attained, in the sense of convergence (5.6), by a sequence of optimal pairs $\left\{\left(A_{\varepsilon, k}^{0}, y_{\varepsilon, k}^{0}\right)\right\}_{\substack{c>0 \\ k \in \mathbb{R}}}$ to problems (4.1)-(4.4).

\section{Acknowledgements}

The research is partially supported by the State Fund for Fundamental Researches, Grant No. F66/14921 and National Academy of Science of Ukraine, Grant No. 2284/16.

\section{References}

[1] Ambrosio, L., Fusco, N., Pallara, D.: Functions of Bounded Variation and Free Discontinuity Problems. Oxford University Press, New York (2000)

[2] Alessandrini, G., Sigalotti, M.: Geometric properties of solutions to the anisotropic $p$-Laplace equation in dimension two. Ann. Acad. Sci. Fen. Mat. 21, 249-266 (2001)

[3] Bergman, D.J., Stroud, D.: Physical properties of macroscopically inhomogeneous media. In: Ehrenreich, H., Turnbull, D. (eds.) Solid State Physics: 
Advances in Research and Applications, vol. 42, pp. 147-269. Academic Press, New York (1992)

[4] Buttazzo, G., Kogut, P.I.: Weak optimal controls in coefficients for linear elliptic problems. Revista Matematica Complutense 24, 83-94 (2011)

[5] Casas, E., Fernandez, L.A.: Optimal control of quasilinear elliptic equations with non differentiable coefficients at the origin. Rev. Mat. Univ. Compl. Madr. 4(23), 227-250 (1991)

[6] Casas, E., Kogut, P.I., Leugering, G.: Approximation of optimal control problems in the coefficient for the $p$-Laplace equation. I. Convergence result. SIAM J. Control Optim. (accepted)

[7] D'Apice, C., De Maio, U., Kogut, O.P.: On shape stability of Dirichlet optimal control problems in coefficients for nonlinear elliptic equations. Adv. Differ. Equ. 15(7-8), 689-720 (2010)

[8] D’Apice, C., De Maio, U., Kogut, O.P.: Optimal control problems in coefficients for degenerate equations of monotone type: Shape stability and attainability problems. SIAM J. Control Optim. 50(3), 1174-1199 (2012)

[9] Horsin, T., Kogut, P.I.: Optimal $L^{2}$-control problem in coefficients for a linear elliptic equation. Math. Control Relat. Fields 5(1), 73-96 (2015)

[10] Kogut, P.I.: On approximation of an optimal boundary control problem for linear elliptic equation with unbounded coefficients. Discrete Contin. Dyn. Syst. Ser. A 34(5), 2105-2133 (2014)

[11] Kogut, P.I., Leugering, G.: Optimal control problems for partial differential equations on reticulated domains. In: Approximation and Asymptotic Analysis. Series: Systems and Control. Birkhäuser, Boston (2011)

[12] Kogut, P.I., Leugering, G.: Matrix-valued L1-optimal control in the coefficients of linear elliptic problems. J. Anal. Appl. (ZAA) 32(4), 433-456 (2012)

[13] Kupenko, O.P., Manzo, R.: Shape stability of optimal control problems in coefficients for coupled system of Hammerstein type. Discrete Contin. Dyn. Syst. Ser. B 20(9), 2967-2992 (2015)

[14] Kupenko, O.P., Manzo, R.: On an optimal $L^{1}$-control problem in coefficients for linear elliptic variational inequality. Abstr. Appl. Anal. 2013, 1-13 (2013)

[15] Levy, O., Kohn, R.V.: Duality relations for non-ohmic composites, with applications to behavior near percolation. J. Stat. Phys. 90, 159-189 (1998)

[16] Lions, J.-L.: Quelques Méthodes de Résolution des problèmes aux limites nonlinéaire. Dunod-Gauthier-Villars, Paris (1969)

[17] Roubíček, T.: Nonlinear Partial Differential Equations with Applications. Birkhäuser, Basel (2013)

[18] Wang, G., Xia, C.: A characterization of the Wulff shape by an overdetermined anisotropic PDE. Arch. Ration. Mech. Anal. 99, 99-115 (2011) 
[19] Xia, C.: On a Class of Anisotropic Problems, Ph.D.-thesis at Albert-LudwigsUniversität Freiburg (2012)

Olha P. Kupenko

Department of System Analysis and Control

National Mining University

Karl Marx Av., 19

Dnipropetrovsk 49005

Ukraine

and

Institute for Applied and System Analysis of

National Technical University of Ukraine "Kiev Polytechnical Institute"

Peremogy Av., 37, Build. 35

Kiev 03056

Ukraine

Rosanna Manzo

Dipartimento di Ingegneria dell'Informazione ed Elettrica e Matematica Applicata Università degli Studi di Salerno

Via Giovanni Paolo II, 132

84084 Fisciano, SA

Italy

e-mail: rmanzo@unisa.it

Received: 20 April 2016.

Accepted: 25 April 2016. 